\title{
A Game Theoretic Approach for Simultaneous Compaction and Equipartitioning of Spatial Data Sets
}

\author{
Upavan Gupta, Student Member, IEEE, and Nagarajan Ranganathan, Fellow, IEEE
}

\begin{abstract}
Data and object clustering techniques are used in a wide variety of scientific applications such as biology, pattern recognition, information systems, etc. Traditionally, clustering methods have focused on optimizing a single metric, however, several multidisciplinary applications such as robot team deployment, ad hoc networks, facility location, etc., require the simultaneous examination of multiple metrics during clustering. In this paper, we propose a novel approach for spatial data clustering based on the concepts of microeconomic theory, which can simultaneously optimize both the compaction and the equipartitioning objectives. The algorithm models a multistep, normal form game consisting of randomly initialized clusters as players that compete for the allocation of data objects from resource locations. A Nash-equilibrium-based methodology is used to derive solutions that are socially fair for all the players. After each step, the clusters are updated using the KMeans algorithm, and the process is repeated until the stopping criteria are satisfied. Extensive simulations were performed on several real data sets as well as artificially synthesized data sets to evaluate the efficacy of the algorithm. Experimental results indicate that the proposed algorithm yields significantly better results as compared to the traditional algorithms. Further, the proposed algorithm yields a high value of fairness, a metric that indicates the quality of the solution in terms of simultaneous optimization of the objectives. Also, the sensitivity of the various design parameters on the performance of our algorithm is analyzed and reported.
\end{abstract}

Index Terms-Equipartitioning, compaction, game theory, clustering, Nash equilibrium.

\section{INTRODUCTION}

$\mathrm{O}$ ВJECT clustering involves grouping of objects into a set of subgroups in such a manner that the similarity measure between the objects within a subgroup is higher than the similarity measure between the objects from other subgroups. Formally, a clustering problem can be defined as an optimization problem with a set of input patterns $X=\left\{x_{1}, \ldots, x_{j}, \ldots, x_{N}\right\}$, a positive integer $K$, a distance measure $\delta$, and a criterion function $J(C, \delta(.,)$.$) on$ $K$-partitions $C=\left\{C_{1}, \ldots, C_{K}\right\}$ of $X$ and $\delta(.,$.$) . Here, x_{j}=$ $\left(x_{j 1}, x_{j 2}, \ldots, x_{j d}\right)^{T} \in \Re^{d}$, where $d$ is the total number of dimensions, and each $x_{j i}$ is a feature in the feature space. The objective of the problem is to partition $X$ into disjoint sets $C_{1}, \ldots, C_{K}(K \leq N)$ such that $J(C, \delta(.,)$.$) is optimized$ (minimized or maximized). The clustering problems in various scientific disciplines such as biology, computer vision and pattern recognition, communications and computer networks, and information systems have specific optimization requirements, and several customized clustering methodologies have been developed to satisfy these application requirements [19], [38]. Most of these methods

- U. Gupta is with the Office of Decision Support, University of South Florida, BEH-245, 4202 E. Fowler Ave, Tampa, FL 33620.

E-mail: ugupta@admin.usf.edu.

- N. Ranganathan is with the Department of Computer Science and Engineering, University of South Florida, ENB-118, 4202 E. Fowler Ave, Tampa, FL 33620. E-mail: ranganat@cse.usf.edu.

Manuscript received 15 June 2008; revised 24 Aug. 2008; accepted 10 Apr. 2009; published online 21 Apr. 2009.

Recommended for acceptance by S. Zhang.

For information on obtaining reprints of this article, please send e-mail to: tkde@computer.org, and reference IEEECS Log Number TKDE-2008-01-0031. Digital Object Identifier 10.1109/TKDE.2009.110. attempt to optimize a single objective that is identified most appropriate in the context of the application.

In recent years, several new disciplines such as search and rescue robotics, ad hoc networks, facility location, multiemergency management, and multicore architectures have evolved. For practical applications, these disciplines require spatial data and object clustering at various levels. However, the clustering requirements are different from the classical application domains, since multiple criteria, which may be conflicting in nature, are required to be optimized simultaneously in such scenarios.

As an example, we can consider an urban multiemergency situation as shown in Fig. 1a. In addition to the emergency response personnel, deployment of several robotic units may be required to perform search and rescue operations in locations where human investigation is difficult [28]. These robotic units would frequently communicate with each other, as well as with the base station, over a wireless ad hoc network. Due to the limited availability of battery power, and high communication overhead, robotic units must be divided into teams to optimize two important objectives: 1) compaction, to minimize power dissipation in intrateam communication, and 2) equipartitioning, to form teams with uniform power distribution, so that the teams do not drop out of the system due to rapid battery exhaustion. These objectives are competitive in nature and need to be optimized in a simultaneous manner. A clustering performed on the basis of a single objective such as compaction would result in the identification of clusters that may not be equipartitioned. One such clustering solution using KMeans algorithm is 


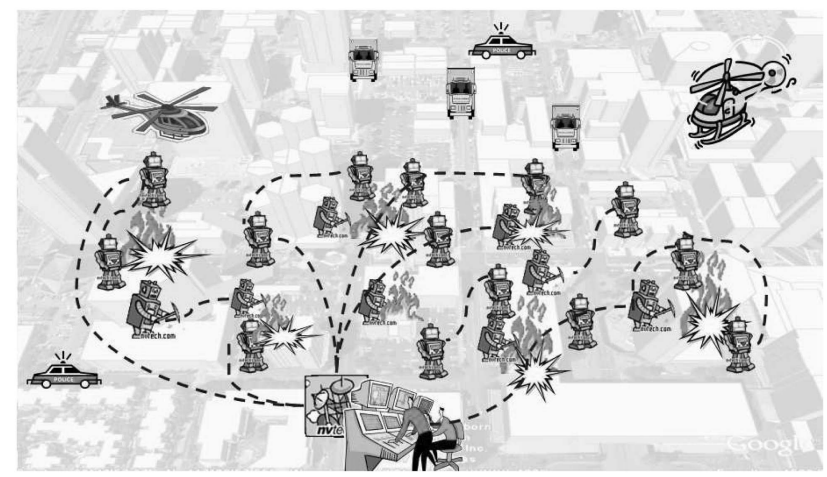

(a)

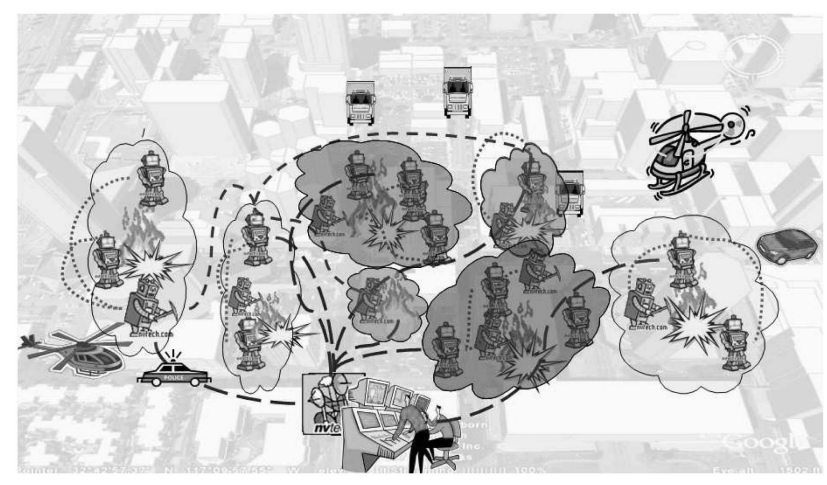

(b)

Fig. 1. An example demonstrating the effect of single-objective clustering to a problem where multiobjective clustering is imperative. (a) Deployment of emergency response units and robotic units for search and rescue operations in an urban multiemergency situation. (b) Clustering of the robotic units to form teams. The clustering performed using the KMeans algorithm identifies clusters such that a few clusters are too large (with five units per cluster), while some of the clusters are too small in size (with one or two units per cluster).

shown in Fig. 1b. As shown, the clusters are not balanced, and the smaller clusters may drop out of the system much earlier than the other clusters due to the consumption of significant amount of power in intercluster communication, in addition to the search and rescue activities.

This entails the investigation of new mechanisms that could perform simultaneous optimization (clustering) on the basis of multiple conflicting objectives. In this research, a Game Theoretic framework for spatial clustering on the basis of multiple conflicting criteria is developed. Specifically, in this paper we have modeled a novel clustering mechanism that performs the optimization on the basis of two conflicting objectives, compaction and equipartitioning, in a simultaneous manner. The algorithm consists of three components: 1) an iterative hill-climbing-based partitioning algorithm, which is utilized to identify initial clusters, 2) a multistep normal form game formulation that identifies the initial clusters as players and resources on the basis of certain properties, and 3) a Nash equilibrium (NE) based solution methodology to evaluate optimal clusters.

The paper is structured as follows: In Section 2, we briefly review the existing clustering methodologies, and the various application domains of game theory. In Section 3, the motivation for identifying a microeconomic clustering approach for new clustering applications is described, and a brief introduction of game theory is presented. The details of microeconomic clustering methodology for simultaneous optimization of compaction and equipartitioning are presented in Section 4 . Also, the complexity of the algorithm, and the applications of the algorithm are discussed in this section. In Section 5, the experimental results for the performance of the algorithm on various real and artificial data sets are presented. Also, the sensitivity analysis, and the quantitative analysis of the fairness of the method are performed. Finally, the conclusions are discussed in Section 6.

\section{Related Work}

Object clustering is a well researched problem, reported extensively in the literature. Several detailed survey papers have reviewed the clustering methods from pattern recognition and image quantization [19], and data mining perspective [3]. Similarly, Murtagh [29] and Baraldi and Blonda [2] have surveyed various hierarchical and fuzzy clustering algorithms, respectively. For a detailed discussion of the various survey papers, one is referred to [38].

Clustering techniques can be classified on the basis of several criteria, such as clustering principles, type of data, shape of clusters, form of final partitions, distance measure, and number of objectives. In this section, the discussion is focused on partitioning of data sets on the basis of clustering objectives. Traditionally, the important clustering objectives have been compaction, connectedness, and spatial separation. The compaction objective attempts to identify clusters with minimum intracluster variation. KMeans algorithm [26] is a simple and widely used mathematical approach in this category. Clustering with an objective of maximization of connectedness ensures that the neighboring data items are clustered together. Density-based methods [8] implement this principle to identify clusters with arbitrary shapes. In spatial-separation-based methods, the objective is to maximize the intercluster separation. However, it provides little guidance during clustering, and may produce trivial results. In addition to these clustering criteria, an important criterion that has received significant attention in the domain of data clustering is equipartitioning or load sharing [10]. Loadsharing methodologies have been widely researched in the field of distributed systems [13]. However, very few clustering techniques that specifically optimize the loadsharing metric are available in literature. The new application domains like ad hoc networks [5], [1], and emergency resource deployment [28] require clusters with almost equal number of data objects per cluster to satisfy the constraints.

From the perspective of clustering methods, in addition to the mathematical clustering techniques, several heuristicsbased algorithms have also been developed. This includes simulated annealing [21], evolutionary algorithms [22], [24], [18], tabu search [11], and ant colony optimization [6]. Also, hybrid approaches that combine multiple algorithms have been proposed in literature [24], [22]. Such techniques are primarily used for feature selection in unsupervised classification, and are largely limited to single-objective optimization. The multiobjective optimization problem has been addressed through the following principles:

1. Ensemble methods. Here, the initial ensembles are created by clustering the data either multiple times using the same algorithm (with different initializations or using bootstrapping) or using complementary 
clustering techniques [36]. The solutions are later combined to create ensembles using expectation maximization or graph-based approaches [36]. However, such a posteriori integration of single-objective clustering results does not exploit the strengths of simultaneous multiobjective optimization.

2. Pareto-optimization. In a pareto-optimization-based method, a feasible solution is pareto-optimal if there exists no other feasible solution that is strictly better. Multiobjective pareto-optimization [18] performs simultaneous optimization of complementary objectives, and hence, identifies better solution points than the ensemble-based methods.

3. Microeconomic methods. An optimization problem with conflicting objectives can be naturally modeled using microeconomic methods, specifically game theory. A problem modeled as a game consists of players with conflicting objectives, competing for receiving resources from a limited supply, in an attempt to optimize their own utilities [9], [32]. The game, when solved using the Nash-equilibrium-based methodology identifies socially fair solution points. The social fairness ensures that every player is satisfied with respect to every other player in the system.

Microeconomic approaches have been applied to a wide spectrum of problems in the domain of computer science. Murugavel and Ranganathan [30] developed auction theoretic algorithms in VLSI design automation for simultaneous gate sizing and buffer insertion problem. In [17], Hanchate and Ranganathan have applied game theoretic concepts for simultaneous optimization of interconnect delay and crosstalk noise through gate sizing, and in [16], Gupta and Ranganathan have implemented game theory for resource allocation and scheduling in the field of multiemergency management. In grid computing, negotiating agents have been used for leasing of resources using such models [23]. Similarly, Grosu and Chronopoulos [12] have used cooperative games and the Nash bargaining solutions for load balancing in distributed systems, and Lazar and Semret [25] have implemented auctions for optimal bandwidth allocation in wired and wireless networks.

In this paper, we identify the multiobjective clustering problem in a normal form noncooperative game theoretic setting. The initial clusters identified using a mathematical approach (KMeans or KMedoids) are modeled as players and resources, different combinations of data objects requested by each player from different resources as strategies, and a function of competing objectives, compaction, and equipartitioning, as the payoff. Since the objectives are convex in nature, as shown in [37] a Nash equilibrium solution always exist, and tries to achieve global optima. Also, depending upon the problem formulation, the complexity of the Nash equilibrium lies between P and NP.

\section{Why Game Theory for Clustering?}

In contrast with the ensemble-based methods that effectively integrate multiple single-objective clustering solutions, the fundamental basis of game theory allows for formulation of problems as multiple interrelated cost metrics competing against one another for optimization. In game theory, each player's decision is based upon the decisions of all other players in the game, and it can optimize its gain with respect to their gains. This results in identification of global gains, and consequently an equilibrium state for the system. As a simple example, in the process of clustering the data objects with the compaction objective, clusters may be identified such that a subset of final clusters would have very few data objects, while another subset would have large number of data objects. This would result in unequal partitions. Alternatively, a clustering performed with load sharing or equipartitioning as an objective may result in the identification of clusters that are suboptimal on compaction objective. These two scenarios are convex in nature, and hence, can be successfully modeled in a game framework. This is the primary motivation for modeling the problem of multiobjective clustering in a game theoretic framework.

The NE for a game theoretic model consists of all the dominant strategies. There may exist multiple NE for a game, and it is possible that some of those NE points may not be pareto-optimal [32]. A good example of such a scenario is the classical example of Prisoners' dilemma [9]. In prisoners' dilemma, the dominant strategy and the NE point is the combination where both the prisoners confess their crimes, which is reasonable from the players' (prisoners') perspective as well as the judiciary system's perspective, considering that the players are rational and noncooperative. It is evident that the solution is pareto-inoptimal. However, paretooptimality in this scenario would require cooperation among the players, existence of a focal arbitrator, and coalition formation, which are infeasible due to multifold increase in the strategy sets for the players, and consequently, the complexity of the game.

A unique property of game theory is social equity or social fairness [37], which ensures that each player in the game is satisfied and the overall goals are reached. In a multiobjective clustering problem with compaction and equipartitioning as the objectives, other optimization methods intend to identify solutions targeting the overall system optimization, rather than the optimization of individual objectives. Instead, a game theoretic modeling ensures that each metric is optimized with respect to the other metrics. For an elaborate discussion on game theory the readers can refer to [9], [32].

\section{Game Theoretic Clustering}

In this section, a detailed description of the proposed game theoretic clustering algorithm is presented. First, a mathematical clustering method is briefly explained, followed by a thorough discussion of the key components of the game theoretic algorithm. Next, an alternative ensemble-based game theoretic method is presented. Finally, the complexity of the algorithm, and the potential applications are discussed in details. Certain assumptions have been made during the modeling of the problem as a game theoretic framework. Most of these assumptions are not restrictive in terms of the applicability of the model, and can be discarded with minor changes. In this work, the algorithm simultaneously models the compaction and equipartitioning metrics. However, other metrics can be incorporated in the model by identifying a convex relationship between the metrics, and defining the strategy, and the payoff functions accordingly. The notations and terminology used in the rest of the paper are given in Table 1. 
TABLE 1

Notations and Terminology

\begin{tabular}{|ll|}
\hline$N$ & Total number of data objects in a data set \\
$d$ & Dimensionality of data set \\
$K$ & Total number of clusters \\
$E_{n k}$ & Euclidean distance between $n$ and $k ; n \in N$ and $k \in K$ \\
$E$ & Sum of the squared Euclidean distance \\
$l_{k}$ & Number of data objects in cluster $k, \forall k \in K$ \\
$l_{\text {ideal }}$ & Number of data objects per cluster in equi-partitioned state \\
& $l_{\text {ideal }=|N / K|}$ \\
$L$ & Sum of the squared load values; $L=\sum_{k=1}^{K}\left(l_{k}-l_{\text {ideal }}\right)^{2}$ \\
$P$ & Total number of players; $P \subset K$ \\
$p_{i}$ & $i^{t h}$ player in a game; $\forall i \in P$ \\
$p_{-i}$ & Set of players in the game other than the player $p_{i}$ \\
$R$ & Total number of resource centers; $R \subset K$ \\
$r_{j}$ & $j^{t h}$ resource center in a game; $\forall j \in R$ \\
$r_{-j}$ & Set of all the resource centers not in the current game \\
$U_{i}$ & Total number of strategies of a player $p_{i}$ \\
$S_{i}$ & Set of all the strategies of player $p_{i}$ \\
$s_{u}^{i}$ & $u^{t h}$ strategy of the player $p_{i} ; s_{u}^{i} \in S_{i}$ and $u=1, \cdots, U$ \\
$S$ & Strategy set of all the strategies in the game \\
& $S=\left\{S_{1}, S_{2}, \cdots, S_{P}\right\}$ \\
$S_{-i}$ & Set of strategy combinations of all players other than $p_{i}$ \\
$s_{v}^{-i}$ & A strategy combination consisting of one strategy \\
& of all players other than $p_{i} ; s_{v}^{-i} \in S_{-i}$ \\
\hline
\end{tabular}

\subsection{Mathematical Partitioning}

KMeans is a simple, yet effective partitioning method for single-objective clustering of a data set of size $N$ into $K$ clusters on the basis of minimization of the total intracluster variation (TICV) or compaction, described as follows:

Let $\left\{\mathbf{x}_{\mathbf{i}}, i=1, \ldots, N\right\}$ be a set of data vectors such that $\mathbf{x}_{\mathbf{i}}=\left\{x_{i 1}, \ldots, x_{i d}\right\}$. Define a boolean $w_{i k}$ for $i=1, \ldots, N$ and $k=1, \ldots, K$.

$$
w_{i k}= \begin{cases}1, & \text { if } i \text { th vector belongs to } k \text { th cluster, } \\ 0, & \text { otherwise. }\end{cases}
$$

Define a matrix $W=\left[w_{i k}\right]$ such that $\sum_{k=1}^{K} w_{i k}=1$, i.e., a data vector can belong to only one cluster (hard partitioning). Now, let $c_{k}=\left(c_{k 1}, \ldots, c_{k d}\right)$ be the centroid of $k$ th cluster, where $c_{k j}$ is given by (2).

$$
c_{k j}=\left(\sum_{i=1}^{N} w_{i k} x_{i j}\right) /\left(\sum_{i=1}^{N} w_{i k}\right) .
$$

Then, the intracluster variation for $k$ th cluster and the TICV based upon the euclidean distance measure is given by (3) and (4), respectively.

$$
\begin{aligned}
& E^{(k)}(W)=\sum_{i=1}^{N} w_{i k} \sum_{j=1}^{d}\left(x_{i j}-c_{k j}\right)^{2}, \\
& E(W)=\sum_{k=1}^{K} \sum_{i=1}^{N} w_{i k} \sum_{j=1}^{d}\left(x_{i j}-c_{k j}\right)^{2} .
\end{aligned}
$$

The objective of KMeans clustering algorithm is to identify clusters that minimize the sum of squared euclidean (SSE) distance measure, and is given as

$$
E\left(W^{*}\right)=\min _{W}\{E(W)\} .
$$

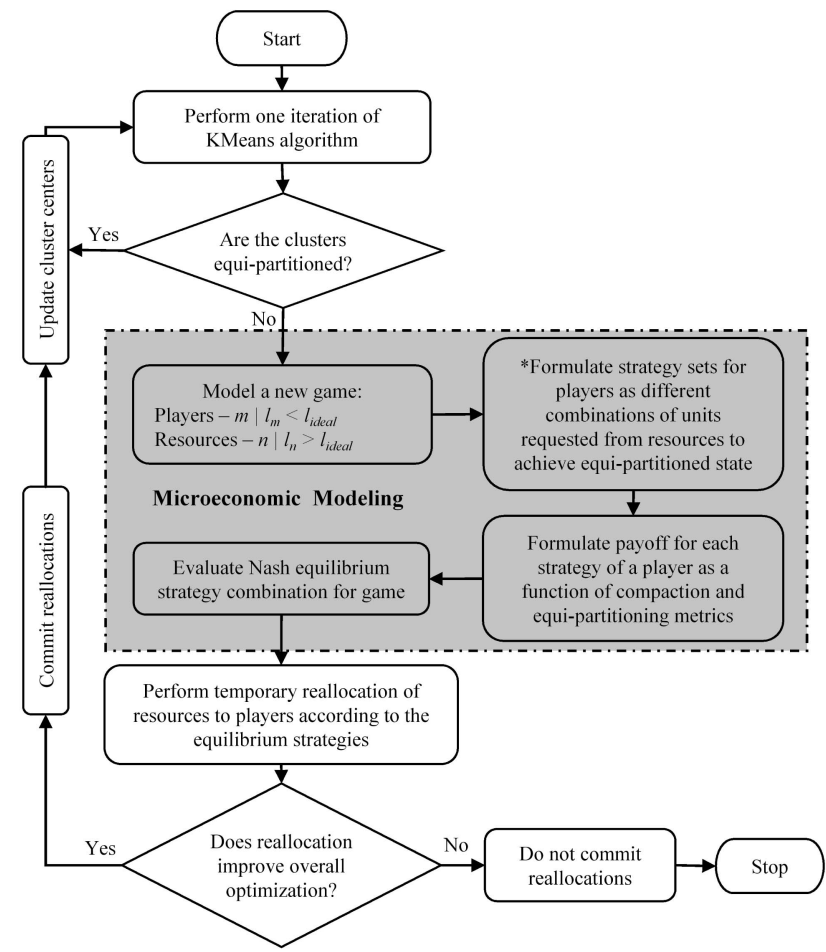

Fig. 2. A flowchart describing the steps involved in the game theoretic clustering algorithm.

The steps involved in the iterative KMeans algorithm are shown in Algorithm 1. The KMeans algorithm is sensitive to the selection of initial cluster heads, and may easily converge to local optima if the choice of initial partitions is improper. However, since KMeans is a fast clustering algorithm, it serves as an efficient initial step for the microeconomic clustering.

Algorithm 1. KMeans Partitioning

Require: K, data set of size $N$ and dimensionality $d$

Ensure: the assignment $w_{n k} \forall n \in N$, where $k \in K$

1: randomly initialize $K$ locations on $d$ dimension space with centroids $c_{k}, \forall k \in K$

2: initialize iteration number $i \leftarrow 0$

3: repeat

4: $\quad i \leftarrow i+1$

5: $\quad$ for $n=1$ to $N$ do

6: $\quad$ calculate $E_{n k}, \forall k \in K$

7: $\quad$ find $k^{\prime}$, such that $E_{n k^{\prime}}=\min \left\{E_{n k}\right\}$

8: $\quad w_{n k^{\prime}}^{i} \leftarrow 1$, and $w_{n k}^{i} \leftarrow 0, \forall k \neq k^{\prime}$

9: $\quad$ end for

10: $\quad$ update $c_{k}$ according to Equation (2), $\forall k \in K$

11: until $w_{n k}^{i}=w_{n k}^{i-1}, \forall n \in N$ and $k \in K$

12: return: $w_{n k} \leftarrow w_{n k}^{i}, \forall n \in N$ and $k \in K$

\subsection{Game Theoretic Clustering}

The overall idea of the game theoretic clustering algorithm is shown in Fig. 2. Initially, a single iteration of KMeans algorithm is performed with random initialization, which identifies initial clusters by minimizing the SSE measure. If the resulting clusters are equipartitioned, then the cluster centers are updated and another iteration of KMeans is performed. However, if the clusters are not equipartitioned, 


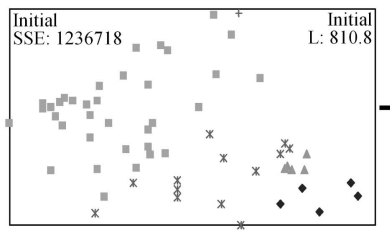

(a)

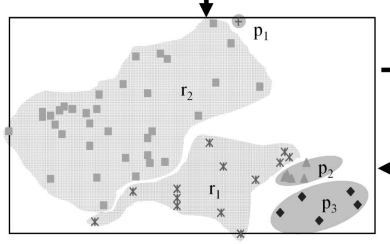

(c)

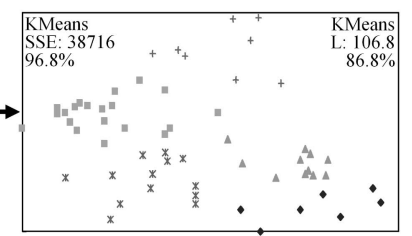

(b)

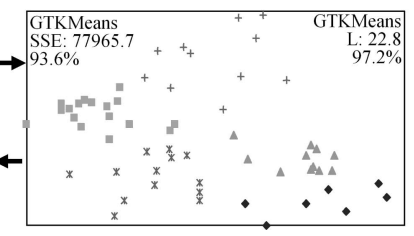

(d)
- Cluster C1 $\quad$ n Cluster C2 $\Delta$ Cluster C3 + Cluster C4 $\quad *$ Cluster C5

Fig. 3. Identification of optimum clusters using game theoretic (GTKMeans) and KMeans methodologies. (a) Initial clusters identified by single iteration of KMeans, (b) final clusters after KMeans, (c) formulation of a game with players $p_{1}, p_{2}$, and $p_{3}$, and resources $r_{1}$ and $r_{2}$, and (d) final clusters after GTKMeans algorithm.

a new game is formulated. The game identifies the players as the clusters that have less than the ideal number of data units. The resources consist of the clusters with more than the ideal size. The ideal size of a cluster is defined as $l_{\text {ideal }}=|N / K|$. Next, the strategy sets for the players are formulated. In the simplest form, the strategy set of a player would consist of the set of different combinations of requested units from resources, such that the player achieves an equipartitioned state. However, we have defined an alternative notion of strategy for this model. The details are described later in this section. Corresponding to each strategy of a player, a payoff or utility is associated. The payoff is a function that models the gain or loss of the player, with respect to other players' strategies. In this model, the payoff is a function of the two objectives, compaction and equipartitioning. Once the payoffs matrices for all the players in the game are formulated, a Nash equilibrium solution is evaluated. The Nash equilibrium strategy set consists of one strategy for each player such that each player is satisfied with respect to all other players in the game. A temporary reallocation of data objects is performed according to the Nash equilibrium strategy set. Next, it is evaluated whether the reallocations improve the overall objective, which is a function of compaction and equipartitioning. If the clusters are optimized, the reallocations are made permanent, and the cluster centers are updated. The procedure is repeated until the stopping criterion is satisfied. The following sections describe the normal form game theoretic model in details.

\subsubsection{Initialization}

The initialization step of the algorithm can be described with the help of an example ${ }^{1}$ given in Fig. 3. During the initialization, cluster centers are randomly generated for the $d$-dimensional data set. This is followed by the identification of initial clusters by performing a single iteration of the

1. The data are taken from German Town Data, which is a twodimensional data set with 59 observations, obtained from [35]. The SSE value for KMeans clustering for five clusters is the reported minimum value in literature [24].
KMeans. As shown in Fig. 3a, the $L$ and the $S S E$ values of the initial clusters are not optimal. If the iterative KMeans, as shown in Algorithm 1, is implemented with the objective of minimization of $S S E$, the final value of the $S S E$ is 38,716 (Fig. 3b). However, the corresponding $L$ value is 106.8, signifying that the clusters are not equipartitioned. Hence, a game theoretic algorithm is required to be formulated with the objective of simultaneous clustering of objects on the basis of compaction and equipartitioning.

The first step in the formulation of the game is to define the components of the game, i.e., the players, the resources, the strategies, and the payoff functions, etc. In the proposed model, the cluster centers with $l_{k}<l_{\text {ideal }}, \forall k \in K$, are identified as the players in the game. Alternatively, the cluster centers with $l_{k}>l_{\text {ideal }}, \forall k \in K$, are considered as the resources in the game. The objective of a player is to receive the data objects from the resources in such a manner that the compaction and equipartitioning objectives are optimized simultaneously. In a situation where multiple players are requesting units from the same resource center, there is a conflict among the players. So, each player competes against all other players in the game in order to maximize its own utility. One such example scenario is displayed in Fig. 3c, where the players $p_{2}$ and $p_{3}$ would compete to receive units from the resource center $r_{1}$.

\subsubsection{Definition of Strategy}

The feasibility of a game theoretic model largely depends upon the notion of strategy, which is an important factor in determining the computational complexity of the model. Essentially, the formulation of the strategies follow a twostep process, where during the first step, the players try to receive resource units from the resource locations that are closest. This minimum euclidean-distance-based allocation is performed irrespective of the requests from other players. However, due to this, a situation may arise where some resource locations may allocate more resources than the overhead available with them. Therefore, for every such resource location, a game needs to be formulated and solved to maintain equipartitioning state. During step two, the cluster centers that have requested resources from the resource location in conflict are considered as the players in a game played specifically for that resource location. The players' strategies in this situation consist of the number of resource units they may have to loose in order to ensure that the corresponding resource location is in consistent, equipartitioned state. An example scenario described in Fig. 4 would be helpful in understanding this notion of strategy.

As shown in Fig. 4, the player $p_{1}$ has requested one resource unit from location $r_{1}$ and player $p_{2}$ has requested four units. Due to these requests, $r_{1}$ may loose five units, which would lead to a situation where $l_{r 1}<l_{\text {ideal }}$. Thus, the players would need to loose a total of three units, and try to receive those units from the resource locations that are farther than $r_{1}$, to ensure that $l_{r 1}=l_{\text {ideal }}$. So, a game is played between the players $p_{1}$ and $p_{2}$, with player $p_{1}$ 's strategy set as $\{0\},\{1\}$, and player $p_{2}$ 's strategy set as $\{0\},\{1\},\{2\}$. The numbers indicate the number of resource units a player may have to loose in order to ensure that the resource center is equipartitioned. The players would receive a payoff for every strategy, which is a function of the additional cost incurred for receiving the resources from resource centers that are farther from the player, and the change in $L$ value for the players and the current resource. Modeling of the strategy in 


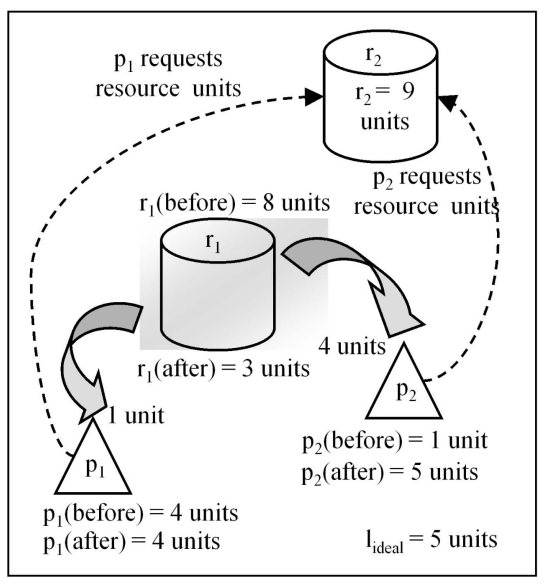

Fig. 4. An example for definition of strategy.

this manner reduces the strategy space considerably. Also, the number of actual players per game is significantly less than the total number of players in the system, since not all players would have requested units from the resource location that is in the conflict situation. Effectively, using this methodology, one large game is subdivided into several smaller games, played in multiple steps.

\subsubsection{The Payoff}

The players in the game play their strategies in order to optimize the equipartitioning and the compaction objectives. An expected utility is associated with each strategy combination of a player in the game. This utility is mathematically modeled as a payoff function, which evaluates the gain or loss a player would incur when it plays its own strategy, and the other players play their corresponding strategies. Algorithm 2 describes the formulation of the payoff function in the context of unified modeling of equipartitioning and compaction objectives. In this model, the payoff for a player $p_{i}$ 's strategy $s_{u}^{i}$, and the players $p_{-i}{ }^{\prime} \mathrm{s}$ strategy combination $s_{v}^{-i}$ for a game played for resource center $r_{j}$ is affected by the following factors:

- Every resource unit that the player intends to loose from $r_{j}$ is received from other resource locations $r_{-j}$, which are farther than $r_{j}$. This increases the SSE value for the player.

- When the other players $p_{-i}$ in the game play $s_{v}^{-i}$ before $p_{i}{ }^{\prime}$ s strategy $s_{u}^{i}$, the cost incurred for receiving the resources from $r_{-j}$ further increases, since some of the closer resource locations might have already allocated data objects to the players $p_{-i}$.

- The equipartitioning metric $l_{r_{j}}$ for $r_{j}$ improves as the players try to receive units from $r_{-j}$. However, as the total number of units lost by the players become greater than $l_{\text {ideal }}$, the equipartitioning value for $l_{r_{j}}$ decreases. Hence, an absolute value of the change in $l_{r_{j}}$ is required to be minimized.

The payoff function captures the interrelationship of the above mentioned criteria, and is modeled as a geometric mean of the total loss incurred by the player $p_{i}$ in terms of the difference between the SSE before and after the other players $p_{-i}$ play their strategies $s_{v}^{-i}$, and the absolute value of the equipartitioning metric $l_{r_{j}}$, corresponding to the strategy $s_{u}^{i}$.

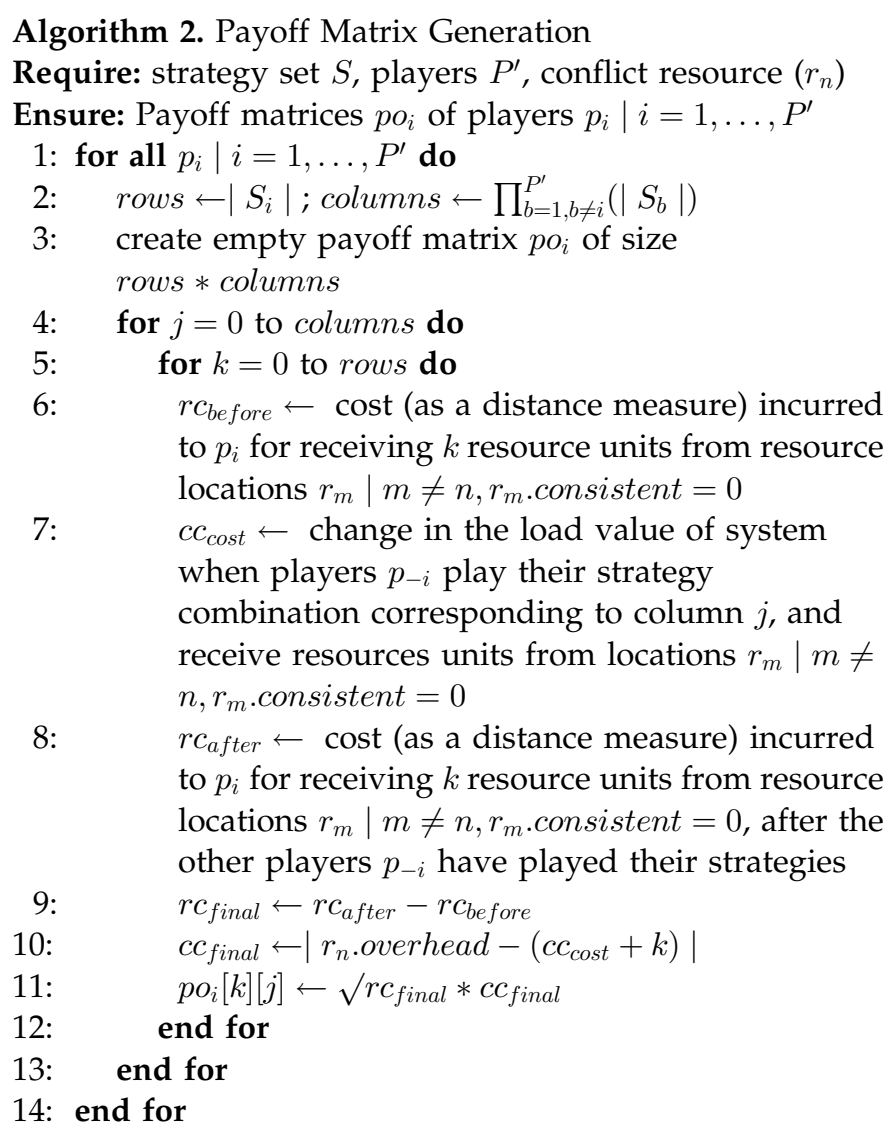

\subsubsection{Nash Equilibrium}

The multiobjective clustering problem being modeled as a game is solved using the Nash equilibrium methodology. As compared to the other solution concepts available in the literature, only Nash equilibrium method identifies social optima. The payoff matrices evaluated during the previous step serve as the input to the algorithm, which generates an output as a Nash equilibrium strategy set consisting of one strategy for each player in the game. At the Nash equilibrium point, no player has an incentive to change its strategy unilaterally. The Nash equilibrium methodology is explained in Algorithm 3.

After the equilibrium strategies are identified, a temporary reallocation of resource units is performed according to the chosen strategies. If the reallocations improve the overall objective, the allocations are committed. The game is then played for other resource locations in conflict and the allocations are performed accordingly. The cluster means are then updated, and the complete process is repeated until there is no further improvement in the objectives.
Algorithm 3. Nash Equilibrium Algorithm
Require: Payoff Matrices $p o_{i}$ of players $p_{i} \mid i=1, \ldots, P^{\prime}$
Ensure: Nash equilibrium strategy combination $S^{*}$
1: for all pay $_{i} \mid i=1, \ldots, P^{\prime}$ do
2: $\quad$ identify a strategy $s_{i}^{*}$ such that
3: $\quad p o_{i}\left(s_{1}, \ldots, s_{i}^{*}, \ldots, s_{P^{\prime}}^{*}\right) \geq p o_{i}\left(s_{1}, \ldots, s_{i}, \ldots, s_{P^{\prime}}^{*}\right)$
4: $\quad / /$ NE Identified on the basis of [31]
5: end for
6: $S^{*}=\left\{s_{1}, \ldots, s_{P^{\prime}}^{*}\right\}$
7: return $S^{*}$ 


\subsection{Ensemble-Based Game Theoretic Clustering}

As shown in the previous section, simultaneous clustering on the basis of multiple objectives is performed using multiple game iterations, where each iteration consists of multistep games. The complexity of this method depends upon the number of data objects as well as the number of clusters, and thus the response time of algorithm may be high for large data sets. Hence, an ensemble-based algorithm that performs clustering on the basis of any mathematical clustering method like KMeans, followed by a game theoretic algorithm has also been proposed in this work. In this method, a complete KMeans clustering is first performed to identify the clusters with best $S S E$ values. The steps of KMeans are explained in the Algorithm 1. The clusters obtained after KMeans algorithm would potentially be inoptimal on the equipartitioning metric. In that case, a game is formulated by modeling players as the clusters with size less than $l_{\text {ideal }}$, and resources as the clusters with size greater than $l_{\text {ideal }}$. The game is then played only once for each conflicting resource center, and a Nash equilibrium solution is identified. A reallocation of the data objects is performed if relative change in the compaction and the equipartitioning values is below a threshold. Only one set of games is played in this scenario. Although, the post KMeans game theoretic algorithm, referred as PKGame henceforth, does not perform simultaneous optimization of multiple objectives, the methodology is fast, and the results obtained during the simulations were promising.

\subsection{Analysis and Application of Algorithm}

In this section, the proposed methodology is analyzed to evaluate its practicability. The computational complexity of the methodology for the extreme cases, as well as the worst case scenario is identified, and a discussion of some of the potential applications of the proposed clustering algorithm is presented.

\subsubsection{Computational Complexity}

In a normal form $P$-player game with an average $S$ strategies per player, the worst-case time complexity is given by $O\left(P * S^{P}\right)$ [31], when the game is played in a single step. However, in the proposed model, a multistep game has been formulated and solved. So, the overall computational complexity of playing $R$ such games is $O\left(R * P * S^{P}\right)$, where $R \subset K, P \subset K, R+P \leq K$, and $K$ is the total number of clusters. Among $R, P$, and $S$, the complexity is largely governed by the value of $S$, which depends upon the definition of a strategy. As opposed to the classical notion of strategy as a combination of resource requests from every resource location, in this work the strategy has been defined as the number of resources a player may have to loose in order to ensure that the resource location is in consistent, equipartitioned state. This restricts the size of strategy set of a player $p_{i}$ as $\left|S_{i}\right|=\lfloor N / K\rfloor$. Hence, the worst-case time complexity of one game is given as $K *\lfloor N / K\rfloor^{K}$, since $P \ll K$.

If there exists only one cluster, i.e., $K=1$, the computational complexity would be $R *\left(1 * N^{1}\right)=R * N$. Similarly, if $K=N$, the complexity would be $N * 1^{N}$, since $l_{\text {ideal }}=1$. Therefore, for the extreme cases, the complexity of the system is $O\left(N^{2}\right) \ll O\left(R * P * S^{P}\right)$. In the worst-case scenario, the number of players in the game are equal to the number of resources in the game $(K=N / 2)$, and the complexity of the system is given by (6).

$$
(N / 2) *(N / 2) *\lfloor N /(N / 2)\rfloor^{N / 2}=N^{2} * 2^{(N-2) / 2} .
$$

The complexity of this algorithm depends primarily on the number of games and the number of data objects in the data set. Hence, the proposed methodology is ideally suited for multiobjective clustering in small to medium sized data sets.

The Nash equilibrium solution points possess certain attributes that make the methodology appropriate for new applications. A Nash solution point is socially equitable, which means that every player in the system is satisfied with respect to every other player, and hence, is in the equilibrium. Social satisfaction is important in the scenarios where every objective in a multiobjective clustering has equal priority. Another important aspect of Nash equilibrium is that for a mixed-strategy noncooperative game, a Nash equilibrium solution point always exists [31]. Although, a pure strategy game has been modeled in this work, the model can be easily extended as a mixed-strategy game by associating probabilities corresponding to the strategies of the players.

\subsubsection{Applications of GTKMeans}

The proposed algorithm is applicable as a clustering methodology for several engineering as well as other optimization problems. One important application of the proposed methodology is in the domain of facility location. In a facility location problem, the resources such as ambulances, distribution depots, and business centers are intended to be located on a terrain on the basis of optimizing either p-center or p-median objectives [39]. In addition to these objectives, load balancing is an important objective for facility location and recent works [14] have tried to optimize both p-center and load balancing problem during facility location. Load balancing is important to ensure that each depot location is profitable and not over loaded as well. The GTKMeans algorithm is directly applicable here to cluster the consumers in the region on the basis of equipartitioning (load balancing) and compaction ( $p$-center) to identify the cluster centers, which effectively serve as the depot locations. GTKMeans can also be used for simultaneous clustering on the basis of $\mathrm{p}$ center and p-median objectives.

As described in Section 1, simultaneous clustering of the robots on the basis of uniform power distribution and compaction is required in the multiemergency search and rescue environments. The state-of-the-art research in robotics [34], [7] has identified the need for such type of clustering methods. A preliminary version of the GTKMeans algorithm has been applied for multirobot team formation [15], where the robots are clustered into teams on the basis of these two objectives. Similarly, in the growing domain of ad hoc and sensor networks, the proposed algorithm is directly applicable to cluster the network nodes. In ad hoc networks, the nodes are partitioned into clusters to minimize the intracluster and intercluster communication overhead [5]. In addition, within each cluster, one node is assigned as the cluster head, which forms the network backbone and is responsible for all intercluster communications for that 
cluster. Now, due to the battery power constraints in ad hoc networks, if a cluster is too large the intracluster point-topoint communication overhead is very high, and the nodes may drop out of the system quickly. Alternatively, if the cluster is too small, the nodes within the cluster will frequently receive the opportunity to be the cluster head, resulting in rapid power dissipation due to long distance communication overhead, thereby dropping out of the system. However, if the clusters are approximately equipartitioned, the performance of the network improves significantly [1]. Using the GTKMeans algorithm, the ad hoc network can be partitioned into load balanced as well as compact partitions. An important aspect of all these applications is that these applications do not consist of very large data sets, and require both objectives to be optimized in a simultaneous manner. Thus, GTKMeans is an appropriate clustering mechanism for these application domains. In addition to these applications, our algorithm can also be applied to solve the problems of circuit placement in VLSI [33], process scheduling in distributed systems, and workload partitioning in multicore computing.

\section{EXPERIMENTAL Results}

Several single-objective clustering methodologies have been developed and employed for various applications. However, in the multiobjective clustering domain, very few methods have been proposed, which significantly limits the comparative study of the performance of the proposed algorithm. Specifically, the only seminal work that performs simultaneous optimization of two performance metrics is an evolutionary-algorithm-based method proposed by Handl and Knowles [18]. However, the algorithm specifically optimizes the compaction and connectedness objectives, and hence cannot be compared with the game theoretic clustering algorithm proposed in this work. Hence, in this section, the performance of the game theoretic algorithm, referred as GTKMeans henceforth, is compared to the KMeans algorithm, and a modified KMeans algorithm emulating the weighted multiobjective optimization methodology.

The first set of experiments were performed with real data sets being used in the previous studies. To analyze the algorithm more closely in terms of efficiency and quality of the solution, artificial data sets were created to simulate the real world scenarios, and the proposed method was exhaustively tested on these data sets. Also, the sensitivity of the proposed algorithm in terms of the various parameters like the number of clusters, the number of data objects per cluster, and the strategy sets of the players has been investigated in this section.

\subsection{Simulation Setup}

The GTKMeans was tested on several data sets that are widely used in the literature for evaluation of general purpose clustering approaches. The data sets are listed as follows:

- British Town Data (BTD). A set consisting of the four principal socioeconomic data components of 50 British towns. The data set was obtained from [4].

- German Town Data (GTD). A two-dimensional data set containing the location coordinates of 59 German towns. The data set was obtained from [35].
The real data sets available in the literature often have an intrinsic structure in terms of the clustering criteria that a specific clustering methodology tries to optimize. Due to this property, the clustering methods that are suitable for certain data sets may not be appropriate for clustering other data sets. Hence, in order to evaluate the performance of the algorithm, and analyze the sensitivity of its various attributes, a wider range of artificial data sets need be constructed. In this work, we have developed two such types of data sets as described below.

- DATA-A: Normally distributed data sets consisting of the location coordinates of data objects on a twodimensional grid of size $12 * 12$ were created. For each data set, the values of mean and variance were varied from $0 \leq \mu \leq 10$ and $\sigma= \pm 2$, respectively, and 704 different data sets were created. The sizes of the data sets were varied from 50 to 150 data objects, partitioned into 3-10 clusters. Also, the intracluster similarity measures in terms of the number of data objects per cluster were taken into consideration. As an example, a data set named 6_8_90 would have 90 data objects partitioned into six clusters, with each cluster having the number data objects ranging from $\lfloor 0.8 *(90 / 6)\rfloor=12$ to $\lfloor(0.2 *(90 / 6))+(90 / 6)\rfloor=15$. For each experiment, an average of 200 repetitions were performed with random cluster center initializations. These data sets were developed to demonstrate the effectiveness of the proposed algorithm for simultaneous clustering on the basis of compaction and equipartitioning.

- DATA-B: To study the performance of the methodologies in optimizing the equipartitioning objective, artificial spatial data sets were developed. In these data sets, equipartitioned clusters were created with different degrees of compaction. On a two dimension grid of size $140 \times 140,150$ data points were partitioned equally into a specified number of clusters with random cluster centers. The radius of each cluster center was varied from 30 to 70 to create data sets that range from compact to coarse, respectively. In effect, 40 such data sets were created by varying the number of clusters from 3 to 10 , and the degree of compaction of clusters varied between 30 and 70 (in quantum of 10).

The Nash equilibrium solution to the n-person normal form game was identified using the Simplical Subdivision algorithm. Among the several Nash equilibrium methodologies available in literature, the simplical subdivision method has been identified to work consistently better than the other methods. The algorithm is acceptably fast for moderate sized problems. Based upon the simplex method, this algorithm starts with a given grid size, and converges to an approximate solution point by iterative labeling of the subsimplexes. GAMBIT [27], an open source C library of game theory analyzer software toolkit was used for the identification of Nash equilibrium solution. Gambit incorporates several Nash equilibrium algorithms for solving normal form, extensive form, and Bayesian games. All experiments were performed on a Sunblade 1,500 workstation that had 4 GB of RAM. 


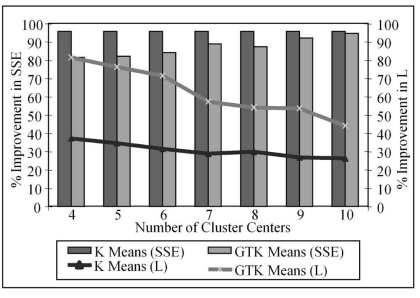

(a)

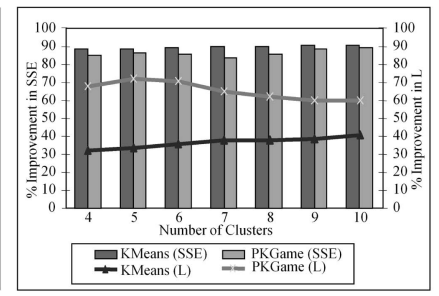

(b)
Fig. 5. Average improvement in the performance for existing data sets. (a) British Town Data. (b) German Town Data.

\subsection{Experiments with Existing Data Sets}

To evaluate the performance of GTKMeans algorithm, it was compared with the KMeans algorithm on the British Town data set [4]. Since KMeans and GTKMeans algorithms have same starting points, and both methods identify same clusters during the initialization phase, the initial knowledge of the environment is same for these methods. Afterward, the KMeans algorithm proceeds with an objective of cluster compaction $(S S E)$, whereas the GTKMeans optimizes the compaction, as well as the equipartitioning measures $(L)$. Fig. 5 a displays a comparative graph for the performance of GTKMeans and KMeans algorithms. The improvement in SSE (Y-axis on left) and $L$ (Y-axis on right) values over the initial clusters for different number of clusters are displayed in the graph. As evident from the graph, for $K=4, \ldots, 10$ the percentage improvement in the $L$ objective for GTKMeans was much higher than corresponding KMeans values, whereas KMeans performed better than GTKMeans on the $S S E$ metric. This is due to the fact that the KMeans algorithm performs a single-objective optimization only on the basis of compaction, while the GTKMeans algorithm identifies clusters by simultaneously optimizing both clustering objectives. For GTKMeans, the average improvements in $S S E$ and $L$ metrics were 87.3 and 62.7 percent, respectively. In case of KMeans, even though the improvement in $S S E$ measure was 95.8 percent, the equipartitioning measure improved by only 30.7 percent. Overall, the GTKMeans algorithm showed an average improvement of 20 percent higher than that of the KMeans algorithm.

Experiments were performed on the German Town data set [35] to evaluate the performance of the post KMeans game theoretic algorithm, called PKGame. The performance of the algorithm in optimizing the two objectives is shown in Fig. 5b. The graph displays the relative performance of the PKGame and the KMeans algorithms. The PKGame algorithm outperformed KMeans in terms of the average improvement in the $L$ for the clusters. However, the $S S E$ metric was largely unaffected, since the KMeans algorithm had already optimized this metric as first step of PKGame algorithm. The average overall improvements in both metrics were 18 percent for the PKGame algorithm.

The experiments on the existing data sets were promising, and demonstrated the potential applicability of the proposed algorithm. Overall, the game-theory-based multimetric clustering method outperformed the KMeans algorithm in terms of simultaneous optimization of multiple

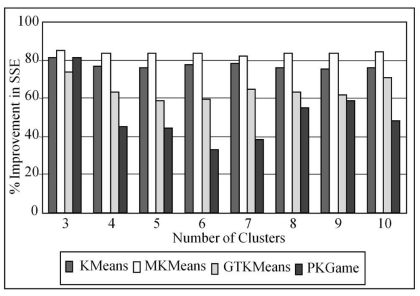

(a)

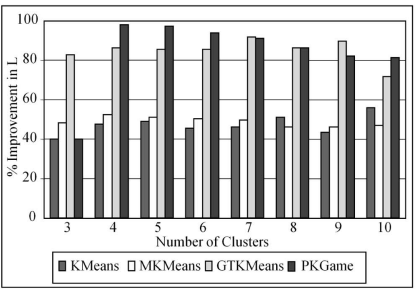

(b)
Fig. 6. Average improvement in the objectives for artificial data sets. (a) Improvement in compaction. (b) Improvement in equipartitioning.

objectives. Although, this algorithm is slower than KMeans in identifying clusters, it provides socially fair solutions. However, a thorough analysis of the algorithm required further experimentation. Hence, simulations were performed on artificial data sets to evaluate the various sensitivity measures, as well as the performance measures of the method.

\subsection{Experiments with Artificial Data}

To evaluate the performance of the proposed microeconomic approaches, multiobjective clustering was performed on the artificial data sets of the types TYPE-A and TYPE-B. The average improvements in the $S S E$ and $L$ metrics were identified for these data sets.

\subsubsection{Experiments with DATA-A}

The comparative analysis between a multiobjective optimization algorithm like GTKMeans, and single-objective optimization algorithm like KMeans advocates the need for multiobjective optimization. However, it does not present a fair performance comparison between the optimization methods. Thus, we compared the GTKMeans algorithm with a modified KMeans algorithm, which incorporates the equipartitioning metric as a clustering objective in addition to the original compaction objective. In this modified KMeans (MKMeans) method, the clustering was performed on the basis of weighted average of the $S S E$, and the $L$ values. The two metrics were equally weighted in order to ensure equal representation in the solution. For the data set DATA-A, the average of the improvements in $S S E$ and $L$ metrics was plotted on graphs shown in Figs. 6a and 6b, respectively. Fig. 6a shows the performance of the algorithms for the compaction objective. The KMeans and MKMeans algorithms performed better than the game theoretic algorithms. This behavior is intuitive as the means-based partitioning methodologies are primarily based on optimizing only the $S S E$ objective. The improvements in the compaction objective for the GTKMeans algorithm are greater than 60 percent for all the cases. From Fig. 6b, it is evident that the performance of KMeans for equipartitioning objective is significantly worse as compared to the GTKMeans and PKGame methods. This follows from the fact that the two objectives are inversely correlated, and an improvement in one objective adversely affects the other objective.

Since the GTKMeans method simultaneously optimizes both the objectives, the clustering performance improved by more than 60 percent on both objectives, as shown in the graphs. Another observation was that the performance of the ensemble-based PKGame method did not follow any 


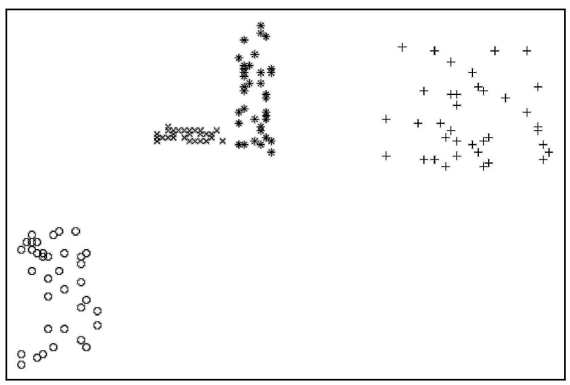

(a)

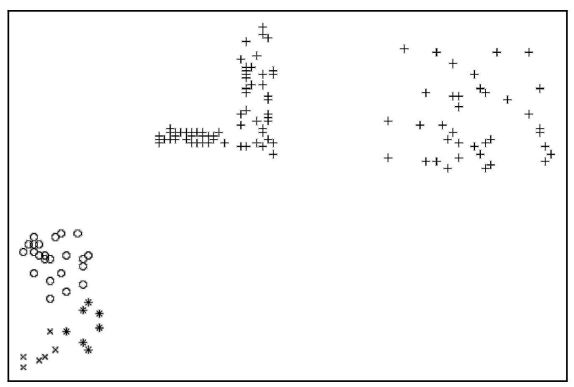

(d)

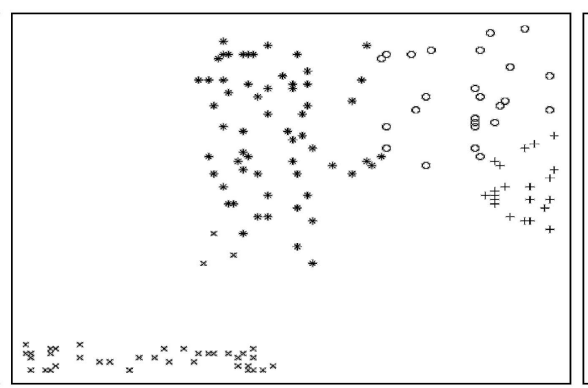

(b)

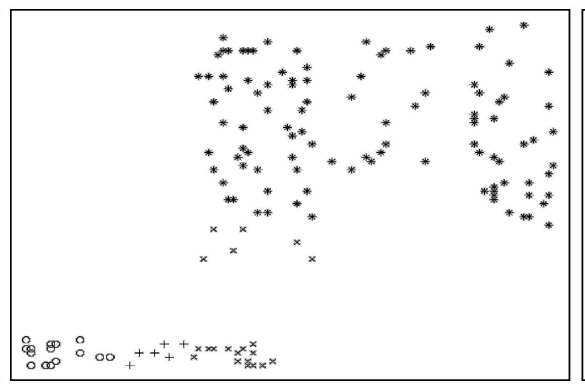

(e)

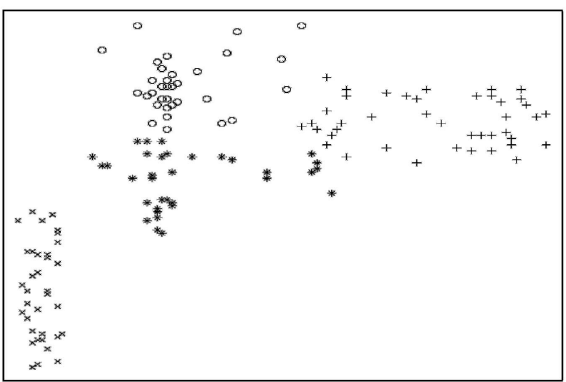

(c)

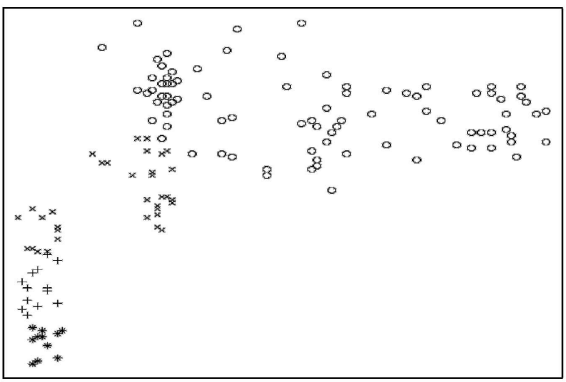

(f)

Fig. 7. Equipartitioning results for four clusters with different degrees of compactness determined by the radius. (a) GTKMeans: Radius $=40$. (b) GTKMeans: Radius $=60$. (c) GTKMeans: Radius $=70$. (d) KMeans: Radius $=40$. (e) KMeans: Radius $=60$. (f) KMeans: Radius $=70$.

specific performance trend. This is attributed to the fact that if the equipartitioning objective was not optimized during the first step, any improvement in the equipartitioning would be significantly higher than the corresponding decrease in the compaction value. This may result in worsening of the compaction objective considerably, which is undesirable in this algorithm. These experiments show that the simultaneous optimization of objectives is an important attribute of a multicriteria clustering technique, which cannot be replaced by sequential optimization of individual metrics.

\subsubsection{Experiments with DATA-B}

Next, the simulations were performed on the artificial data sets, DATA-B, developed specifically to evaluate the performance of the algorithms on the optimization of the equipartitioning objective. In DATA-B, each data set consisted of data points on a two-dimensional grid, with randomly initialized cluster centers. Each cluster center (an $x-y$ location) had a predefined radius which determined the compactness of the cluster, which was same for all the clusters in a particular data set. The data objects were randomly generated points on the grid such that each point was located within the radius of its corresponding cluster center. The data objects were equally distributed among the total number of clusters in the data set to ensure that the ground truth was equipartitioned. Clustering was performed on these data sets using the KMeans and the GTKMeans algorithms with random initialization of cluster centers, and the results were evaluated for their performance on the equipartitioning and the compaction objectives. The clustering results shown in Figs. 7 and 8 correspond to the best case results for GTKMeans and KMeans clustering algorithms for partitioning the data into four and six clusters, respectively.
For different radii, the clustering performances of the algorithms are shown.

It is apparent from Figs. 7 and 8 that our algorithm outperforms the KMeans algorithm on the equipartitioning objective. Also, as the radius increases, the intercluster separation decreases, resulting in superior performance of the GTKMeans algorithm over the KMeans algorithm. This improvement can be seen clearly in Figs. $7 \mathrm{~b}$ and $7 \mathrm{e}$ for radius $=60$, and in Figs. 7c and $7 \mathrm{f}$ for radius $=70$, respectively. Another important observation is that the clustering performance of the GTKMeans improves as the number of clusters increases. With the increase in the number of clusters and the radius of each cluster, the separation between the adjoining clusters is very small, and hence the performance of the KMeans algorithm is adversely affected. In some cases, for the KMeans algorithm a subset of clusters is empty whereas some of the clusters are too large. Such data sets have extremely high $L$ values. One such example is shown in Fig. 8f.

The average performance of the two algorithms on the data set DATA-B is shown in Table 2. As shown in the table, the GTKMeans algorithm performs significantly better than the KMeans algorithm on average. Not only the optimization results for $L$ metric are multifold better, the optimization of the $S S E$ metric is also comparable to the KMeans algorithm.

\subsection{Fairness}

Identification of socially fair solutions by optimizing each objective with equal priority, is an important attribute and strength of the game theoretic models. To appropriately evaluate the social fairness of the proposed algorithms, a quantitative measure of the fairness of the algorithms in optimizing $S S E$ and $L$ must be identified. Among the various models, Jain's Fairness Index [20], and geometric mean index 


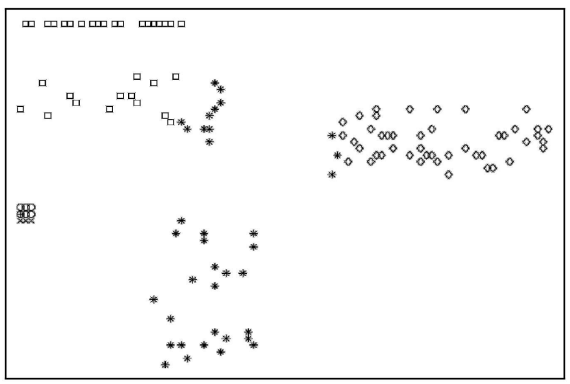

(a)

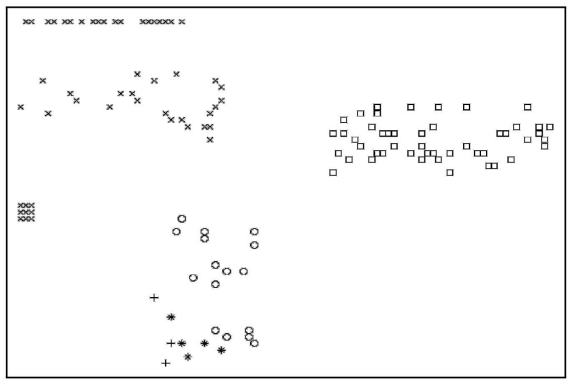

(d)

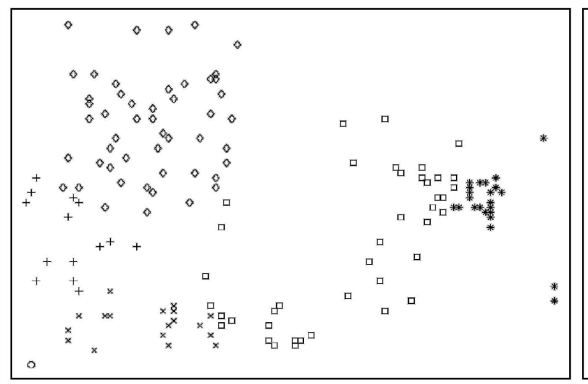

(b)

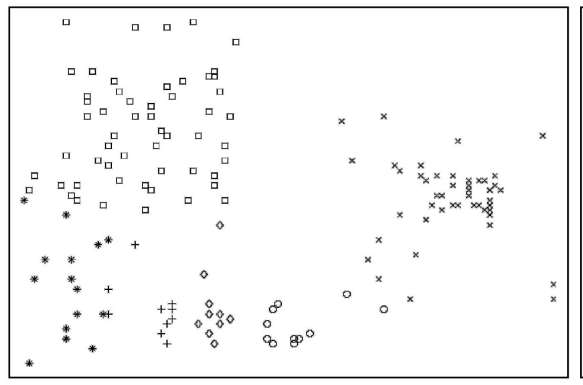

(e)

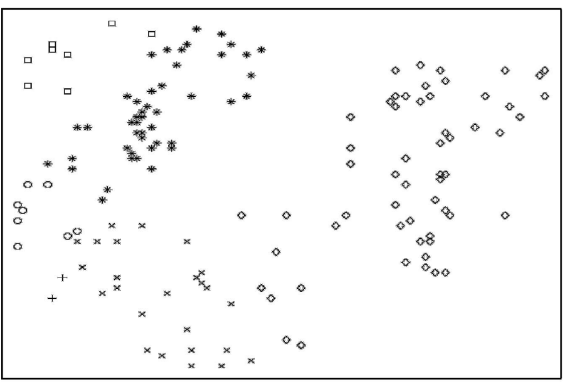

(c)

(f)

Fig. 8. Equipartitioning results for six clusters with different degrees of compactness determined by the radius. (a) GTKMeans: Radius $=40$. (b) GTKMeans: Radius $=50$. (c) GTKMeans: Radius $=60$. (d) KMeans: Radius $=40$. (e) KMeans: Radius $=50$. (f) KMeans: Radius $=60$.

are two appropriate criteria. According to the Jain's index, the fairness of the methodology is identified using (7).

$$
\text { fairness }=\left(\sum_{i=1}^{n} x_{i}\right)^{2} /\left(n * \sum_{i=1}^{n} x_{i}^{2}\right) .
$$

Here, $x_{i}$ corresponds the improvement in the $i$ th objective. The fairness value ranges from 0 (worst case) to 1 (best case). Similarly, the geometric mean index identifies the relative improvements in optimization values of the various clustering criteria as a single index. Table 3 shows the fairness values for different number of clusters. As shown, the GTKMeans method has a high Jain's fairness index averaging 0.98 as compared to the KMeans value of 0.93 (DATA-A). This signifies that the GTKMeans method optimizes both the objectives with almost equal priority. Similarly, the geometric mean index value of the GTKMeans is higher than the KMeans by more than 15 percent. The fairness performance of the MKMeans and the PKGame methods are also inferior to the GTKMeans.

\subsection{Sensitivity Analysis}

The experiments performed on the artificial data sets provided indications about the sensitivity of various attributes of the proposed game theoretic model, on the clustering performance. In this section, a quantitative analysis of the sensitivity of number of players, number of strategies per game, response time of the algorithm, and structure of the data set is presented. These attributes significantly affect the practicability of the algorithm as a viable clustering method.

\subsubsection{Data Set Similarity Measure}

The structure of the data set has a notable impact on the performance of an algorithm. The similarity measure of a

TABLE 2

Performance Comparison of Algorithms for DATA-B

\begin{tabular}{|c|c|c|c|c|c|c|}
\hline \multirow[t]{2}{*}{ Data Set干 } & \multicolumn{2}{|c|}{$\%$ Improvement GTKMeans $\dagger$} & \multicolumn{2}{|c|}{ \% Improvement KMeans $\ddagger$} & \multicolumn{2}{|c|}{ Avg. \% Improvement in Objectives } \\
\hline & Equi-Partitioning & Compaction & Equi-Partitioning & Compaction & GTKMeans & Kmeans \\
\hline $3-150$ & 258.6 & 128.7 & 74.6 & 189.7 & 193.7 & 132.2 \\
\hline $4-150$ & 2286.4 & 355.5 & 168.3 & 534.4 & 1321.0 & 351.4 \\
\hline $5-150$ & 1142.2 & 412.5 & 311.3 & 600.8 & 777.4 & 456.1 \\
\hline $6-150$ & 1646.6 & 449.2 & 192.2 & 618.5 & 1047.9 & 405.4 \\
\hline $7-150$ & 1886.6 & 362.7 & 213.2 & 509.8 & 1124.7 & 361.5 \\
\hline $8-150$ & 676.5 & 277.4 & 169.8 & 418.5 & 477.0 & 294.2 \\
\hline $9-150$ & 749.2 & 269.6 & 118.8 & 445.3 & 509.4 & 282.1 \\
\hline $10-150$ & 581.8 & 279.5 & 266.8 & 435.1 & 430.7 & 351.0 \\
\hline & & & & Average & 735.2 & 329.2 \\
\hline
\end{tabular}


TABLE 3

Fairness of the Clustering Algorithms

\begin{tabular}{|c|c|c|c|c|c|c|c|c|c|c|c|c|c|c|c|c|c|}
\hline Clustering & \multicolumn{10}{c|}{ Geometric Mean Index } & \multicolumn{1}{c|}{ Jain's Fairness Index } \\
Algorithm & 3 & 4 & 5 & 6 & 7 & 8 & 9 & 10 & 3 & 4 & 5 & 6 & 7 & 8 & 9 & 10 \\
\hline KMeans & 57.1 & 60.5 & 61.2 & 59.4 & 60.1 & 60.9 & 56.9 & 62.0 & 0.89 & 0.94 & 0.95 & 0.93 & 0.93 & 0.95 & 0.93 & 0.96 \\
\hline MKMeans & 64.0 & 65.9 & 65.2 & 64.9 & 63.7 & 62.2 & 62.0 & 62.7 & 0.92 & 0.94 & 0.94 & 0.94 & 0.94 & 0.92 & 0.92 & 0.92 \\
\hline GTKMeans & 78.0 & 73.8 & 72.7 & 71.2 & 77.1 & 73.8 & 74.3 & 76.2 & 0.90 & 0.98 & 0.98 & 0.97 & 0.97 & 0.98 & 0.97 & 0.99 \\
\hline PKGame & 57.1 & 66.5 & 56.7 & 46.3 & 36.5 & 41.3 & 41.8 & 44.9 & 0.90 & 0.88 & 0.80 & 0.72 & 0.66 & 0.72 & 0.74 & 0.78 \\
\hline
\end{tabular}

data set (DATA-A), which corresponds to the number of data objects per cluster determines the structure of the data set. From the artificial data with variance as $\sigma= \pm 2$, various data sets were generated with different degrees of similarity measures. The effect of structure on the execution time of the algorithm for different similarity measures and cluster sizes is shown in Fig. 9a. As shown, the similarity measure does not impact the performance of the algorithm significantly, i.e., on average, the execution time of the GTKMeans algorithm is independent of the structure of the data set. Hence, it is suitable as a general spatial clustering methodology. The average performance in terms of fairness of allocation is shown in Table 3. The geometric mean fairness is in range 60-80 percent, which is a good measure of fairness. Hence, the structure of a data set does not adversely affect the performance of the proposed methodology.

\subsubsection{Number of Players and Strategies}

An important consideration during the modeling of a problem in a game theoretic framework is the impact of the size of game. The size determines the complexity, and consequently the performance of the system. Thus, the average size of the game in terms of the number of players and the strategies for different clusters was evaluated. The graph shown in Fig. $9 \mathrm{~b}$ displays the range of players and strategies for different number of clusters. An important observation is that although the average number of players increases as the cluster size increases, the total number of players is significantly less than half of the total number of clusters, which is the worst case scenario. For example, on average, there are at most 3.5 players for the simulations with nine clusters. It is also important to note that the average strategy size does not increase exponentially as a function of the number of players, which is frequently an issue in the classical game theoretic models. This behavior is attributed to the novel definition of players and strategies in the proposed model. This modeling reduces the complexity of the system significantly. However, the surge in the number of strategies for data sets with large number of clusters indicate that the GTKMeans is better suited for multiobjective clustering of medium sized data sets with a lower number of clusters per data set.

\subsubsection{Execution Time}

The multiobjective clustering methodology proposed in this work is slower than the KMeans method by multiple orders of magnitude. Similar is the case with other heuristics-based methodologies. In order to quantify the effect of number clusters on the execution time of the algorithm, and analyze the performance extremes, the average execution time and the maximum execution time for different number of clusters (for DATA-A) was plotted. As shown in Fig. 9c, for smaller number of clusters, i.e., $K=3, \ldots, 8$, the GTKMeans performed well and identified the optimal clusters within 10 seconds. Also, the worst case performance followed similar trend. However, for larger number of clusters, the performance decayed exponentially. This is due to the fact that as the number of clusters increase, the potential number of players, and consequently the strategies increase significantly, and the game becomes large. The time complexity of the Nash equilibrium algorithm is high, which results in slower execution time in such cases.

\section{Conclusions}

A novel microeconomic-theory-based technique for simultaneous multiobjective clustering on the basis of two important metrics, compaction, and equipartitioning, has been developed in this research. It models the problem as a hybrid approach involving KMeans and noncooperative

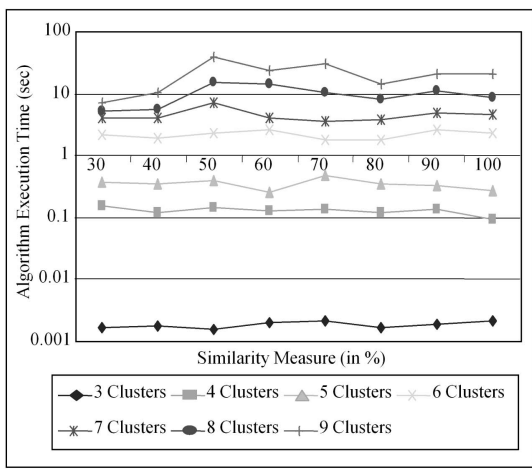

(a)

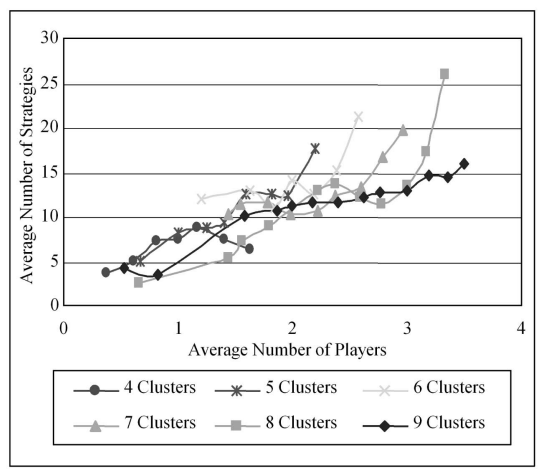

(b)

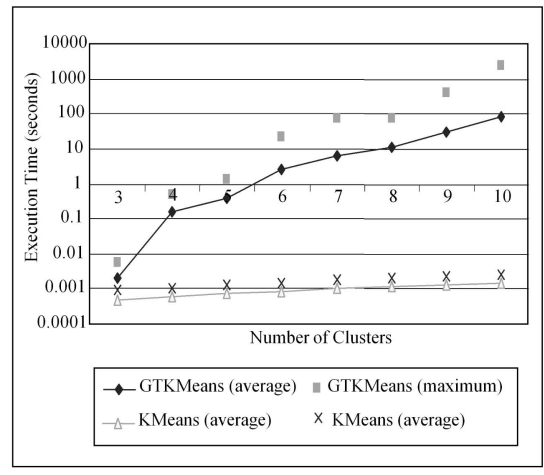

(c)

Fig. 9. Sensitivity analysis of the algorithm. (a) Effect of data set similarity measure on the execution time of algorithm. (b) Average number of players and strategies for different cluster sizes. (c) Relationship between the execution time and the number of clusters. 
multiplayer normal form game with Nash-equilibriumbased solution. Also, a post KMeans game theoretic technique has been proposed in this work. This algorithm performs game theoretic clustering after the complete execution of KMeans algorithm. The experimental study on existing and artificial data sets provided important insights related to the performance of the game theoretic algorithm. As compared to the KMeans, this algorithm performs significantly better in terms of the fairness toward improving the clustering criteria. Also, the complexity of the algorithm in terms of players and strategies has been reduced significantly by developing novel definitions for the players, and the strategies. This algorithm is not sensitive to the structure of the data set. However, being a heuristics-based method, it is slower than the KMeans algorithm, and suitable for clustering of medium sized data sets. Overall, the proposed algorithm is well suited for clustering problems where the objective functions are complementary and need to be optimized simultaneously.

\section{REFERENCES}

[1] A. Amis and R. Prakash, "Load-Balancing Clusters in Wireless Ad Hoc Networks," Proc. Third IEEE Symp. Application-Specific Systems and Software Eng. Technology, pp. 25-32, 2000.

[2] A. Baraldi and P. Blonda, "A Survey of Fuzzy Clustering Algorithms for Pattern Recognition. II," IEEE Trans. Systems, Man and Cybernetics, Part B, vol. 29, no. 6, pp. 786-801, Dec. 1999.

[3] P. Berkhin, "Survey of Clustering Data Mining Techniques," technical report, Accrue Software, vol. 10, pp. 92-1460, 2002.

[4] Y. Chien, Interactive Pattern Recognition. M. Dekker, 1978.

[5] M. Demirbas, A. Arora, V. Mittal, and V. Kulathumani, "A FaultLocal Self-Stabilizing Clustering Service for Wireless Ad Hoc Networks," IEEE Trans. Parallel and Distributed Systems, vol. 17, no. 9, pp. 912-922, Sept. 2006.

[6] M. Dorigo, G. Caro, and L. Gambardella, "Ant Algorithms for Discrete Optimization," Artificial Life, vol. 5, no. 2, 137-172, 1999.

[7] R. Emery-Montemerlo, G. Gordon, J. Schneider, and S. Thrun, "Game Theoretic Control for Robot Teams," Proc. IEEE Int'l Conf. Robotics and Automation (ICRA '05), pp. 1163-1169, 2005.

[8] M. Ester, H. Kriegel, J. Sander, and X. Xu, "A Density-Based Algorithm for Discovering Clusters in Large Spatial Databases with Noise," Proc. Second Int'l Conf. Knowledge Discovery and Data Mining, pp. 226-231, 1996.

[9] F. Forgó, J. Szép, and F. Szidarovszky, Introduction to the Theory of Games: Concepts, Methods, Applications. Kluwer Academic Publishers, 1999.

[10] W. Gale, S. Das, and C. Yu, "Improvements to an Algorithm for Equipartitioning," IEEE Trans. Computers, vol. 39, no. 5, pp. 706710, May 1990.

[11] F. Glover, "Future Paths for Integer Programming and Artificial Intelligence," Computers E Operations Research, vol. 13, pp. 533-549, 1986.

[12] D. Grosu and A. Chronopoulos, "A Game-Theoretic Model and Algorithm for Load Balancing in Distributed Systems," Proc. Parallel and Distributed Processing Symp., pp. 146-153, 2002.

[13] D. Grosu and A. Chronopoulos, "Algorithmic Mechanism Design for Load Balancing in Distributed Systems," IEEE Trans. Systems, Man and Cybernetics, Part B, vol. 34, no. 1, pp. 77-84, Feb. 2004.

[14] S. Guha, A. Meyerson, and K. Munagala, "Hierarchical Placement and Network Design Problems," Proc. 41st Ann. Symp. Foundations of Computer Science, pp. 603-612, 2000.

[15] U. Gupta and N. Ranganathan, "A Microeconomic Approach to Multi-Robot Team Formation," Proc. IEEE/RSJ Int'l Conf. Intelligent Robots and Systems, pp. 3019-3024, 2007.

[16] U. Gupta and N. Ranganathan, "Multievent Crisis Management Using Noncooperative Multistep Games," IEEE Trans. Computers, vol. 56, no. 5, pp. 577-589, May 2007.

[17] N. Hanchate and N. Ranganathan, "Simultaneous Interconnect Delay and Crosstalk Noise Optimization through Gate Sizing Using Game Theory," IEEE Trans. Computers, vol. 55, no. 8, pp. 1011-1023, Aug. 2006.
[18] J. Handl and J. Knowles, "Evolutionary Multiobjective Clustering," Proc. Eighth Int'l Conf. Parallel Problem Solving from Nature, pp. 1081-1091, 2004.

[19] A.K. Jain, M.N. Murty, and P.J. Flynn, "Data Clustering: A Review," ACM Computing Surveys, vol. 31, no. 3, pp. 264-323, 1999.

[20] R. Jain, D. Chiu, and W. Hawe, "A Quantitative Measure of Fairness and Discrimination for Resource Allocation in Shared Computer System," DEC-TR-301, Eastern Research Lab, Digital Equipment Corporation, Sept. 1984.

[21] S. Kirkpatrick, C. Gelatt Jr., and M. Vecchi, "Optimization by Simulated Annealing," Science, vol. 220, no. 4598, pp. 671-680, 1983.

[22] K. Krishna and M. Murty, "Genetic K-Means Algorithm," IEEE Trans. Systems, Man and Cybernetics, Part B, vol. 29, no. 3, pp. 433439, June 1999.

[23] Y. Kwok, S. Song, and K. Hwang, "Selfish Grid Computing: Game-Theoretic Modeling and NAS Performance Results," Proc. Int'l Symp. Cluster Computing and the Grid (CCGrid), 2005.

[24] M. Laszlo and S. Mukherjee, "A Genetic Algorithm Using HyperQuadtrees for Low-Dimensional K-Means Clustering," IEEE Trans. Pattern Analysis and Machine Intelligence, vol. 28, no. 4, pp. 533-543, Apr. 2006.

[25] A. Lazar and N. Semret, "A Resource Allocation Game with Application to Wireless Spectrum," technical report, Columbia Univ., 1996.

[26] J. MacQueen, "Some Methods for Classification and Analysis of Multivariate Observations," Proc. Fifth Berkeley Symp. Math. Statistics and Probability, vol. 1, pp. 281-297, 1967.

[27] R. McKelvey, A. McLennan, and T. Turocy, "Gambit: Software Tools for Game Theory," http://gambit.sourceforge.net, The Gambit Project, 2002.

[28] R. Murphy, "Human-Robot Interaction in Rescue Robotics," IEEE Trans. Systems, Man, and Cybernetics: Part C: Applications and Rev., vol. 34, no. 2, pp. 138-153, May 2004.

[29] F. Murtagh, "A Survey of Recent Advances in Hierarchical Clustering Algorithms," Computer J., vol. 26, no. 4, pp. 354-359, 1983.

[30] A. Murugavel and N. Ranganathan, "A Game Theoretic Approach for Power Optimization During Behavioral Synthesis," IEEE Trans. Very Large Scale Integration Systems, vol. 11, no. 6, pp. 1031-1043, Dec. 2003.

[31] J. Nash Jr., "Equilibrium Points in N-person Games," Proc. Nat'l Academy of Sciences USA, vol. 36, no. 1, pp. 48-49, 1950.

[32] E. Rasmusen, Games and Information: An Introduction to Game Theory. Blackwell Publishers, 2001.

[33] S. Saha, S. Sur-Kolay, S. Bandyopadhyay, and P. Dasgupta, "Multiobjective Genetic Algorithm for K-Way Equipartitioning of a Point Set with Application to CAD-VLSI," Proc. Ninth Int'l Conf. Information Technology, pp. 281-284, 2006.

[34] N. Sato, F. Matsuno, T. Yamasaki, T. Kamegawa, N. Shiroma, and H. Igarashi, "Cooperative Task Execution by a Multiple Robot Team and Its Operators in Search and Rescue Operations," Proc. IEEE/RSJ Int'l Conf. Intelligent Robots and Systems (IROS '04), vol. 2, 2004.

[35] H. Spath, Cluster Analysis Algorithms for Data Reduction and Classification of Objects. Ellis Horwood, 1980.

[36] A. Topchy, A. Jain, and W. Punch, "Clustering Ensembles: Models of Consensus and Weak Partitions," IEEE Trans. Pattern Analysis and Machine Intelligence, vol. 27, no. 12, pp. 1866-1881, Dec. 2005.

[37] A. Vetta, "Nash Equilibria in Competitive Societies, with Applications to Facility Location, Traffic Routing and Auctions," Proc. 43rd Ann. IEEE Symp. Foundations of Computer Science, pp. 416-425, 2002.

[38] R. Xu and D. Wunsch, "Survey of Clustering Algorithms," IEEE Trans. Neural Networks, vol. 16, no. 3, pp. 645-678, May 2005.

[39] A. Zarnani, M. Rahgozar, C. Lucas, and F. Taghiyareh, "Spatial Data Mining for Optimized Selection of Facility Locations in FieldBased Services," Proc. IEEE Symp. Computational Intelligence and Data Mining, pp. 734-741, 2007. 


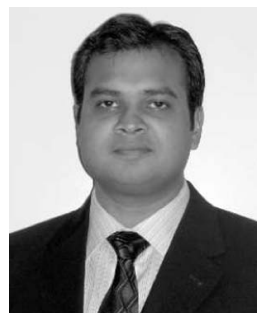

Upavan Gupta received the bachelor's (honors) degree in computer applications from the International Institute of Professional Studies, Indore, India, in 2002, the MS degree in computer science and engineering from the University of South Florida, Tampa, in 2004, and the PhD degree in computer science and engineering from the University of South Florida, Tampa, in 2008. He is currently working as a specialist, computer research, in the Decision Support Systems group of the Office of Provost and senior vice-president at the University of South Florida, Tampa. His research interests include the exploration and development of fast and fair multimetric optimization algorithms for pattern recognition, data mining, search, and rescue robotics, and homeland security applications, variation aware VLSI design automation, and utilitarian optimization algorithms. He is serving on the technical program committee of the IEEE Computer Society International Symposium on VLSI (ISVLSI). He has received the USF Graduate School Outstanding Dissertation Award for the year 20072008 , and is a recipient of the IEEE Computer Society R.E. Merwin Scholarship in 2004. He is a student member of the IEEE.

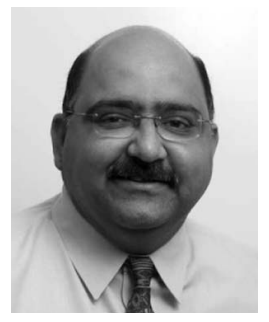

Nagarajan "Ranga" Ranganathan (S'81-M'88$S M$ '92-F'02) received the $\mathrm{BE}$ (honors) degree in electrical and electronics engineering from the Regional Engineering College, National Institute of Technology, Tiruchirapalli, University of Madras, India, 1983, and the PhD degree in computer science from the University of Central Florida, Orlando, in 1988. He is a distinguished university professor of computer science and engineering at the University of South Florida, Tampa. During 1998-1999, he was a professor of electrical and computer engineering at the University of Texas at El Paso. His research interests include VLSI circuit and system design, VLSI design automation, multimetric optimization in hardware and software systems, biomedical information processing, computer architecture, and parallel computing. He has developed many special purpose VLSI circuits and systems for computer vision, image and video processing, pattern recognition, data compression, and signal processing applications. He has coauthored more than 225 papers in refereed journals and conferences, four book chapters, and co-owns six US patents and two pending. He has edited three books titled VLSI Algorithms and Architectures: Fundamentals and VLSI Algorithms and Architectures: Advanced Concepts, IEEE CS Press, 1993, VLSI for Pattern Recognition and Artificial Intelligence, World Scientific Publishers, 1995, and coauthored a book titled Low Power High Level Synthesis for Nanoscale CMOS Circuits, Springer, June 2008. He was elected as a fellow of the IEEE in 2002 for his contributions to algorithms and architectures for VLSI systems. He is a member of the IEEE Computer Society, the IEEE Circuits and Systems Society, and the VLSI Society of India. He has served on the editorial boards for the journals: Pattern Recognition (1993-1997), VLSI Design (1994-present), IEEE Transactions on VLSI Systems (1995-1997), IEEE Transactions on Circuits and Systems (1997-1999), IEEE Transactions on Circuits and Systems for Video Technology (1997-2000), and ACM Transactions on Design Automation of Electronic Systems (2007-2009). He was the chair of the IEEE Computer Society Technical Committee on VLSI during 19972001. He served on the steering committee of the IEEE Transactions on VLSI Systems during 1999-2001, the steering committee chair during 2002-2003 and the editor-in-chief for two consecutive terms during 2003-2007. He served as the program cochair for ICVLSID '94, ISVLSI '96, ISVLSI '05, and ICVLSID '08, and as a general cochair for ICVLSID '95, IWVLSI '98, ICVLSID '98, ISVLSI '05, and ISVLSI '09. He has served on technical program committees of international conferences including ICCD, ICPP, IPPS, SPDP, ICHPC, HPCA, GLSVLSI, ASYNC, ISQED, ISLPED, CAMP, ISCAS, MSE, and ICCAD. He received the USF Outstanding Research Achievement Award in 2002, the USF President's Faculty Excellence Award in 2003, the USF Theodore-Venette Askounes Ashford Distinguished Scholar Award in 2003, the SIGMA XI Scientific Honor Society Tampa Bay Chapter Outstanding Faculty Researcher Award in 2004, and the Distinguished University Professor honorific title and the university gold medallion honor in 2007. He was a corecipient of the Best Paper Awards at the International Conference on VLSI Design in 1995, 2004, and 2006, and the IEEE Circuits and Systems Society Transactions on VLSI Systems in 2009.

$\triangleright$ For more information on this or any other computing topic, please visit our Digital Library at www.computer.org/publications/dlib. 